\title{
Illness behaviour in patients with arthritis
}

\author{
M J Ahern, A C McFarlane, A Leslie, J Eden, P J Roberts-Thomson
}

\begin{abstract}
Objectives-To determine if there are specific patterns of illness behaviour in patients with arthritis, and if abnormal patterns of illness behaviour are associated with withdrawal from trials of antiinflammatory drugs, and to examine which aspects of illness behaviour are perceived by rheumatologists to be related to the disease process.
\end{abstract}

Methods-The illness behaviour questionnaire (IBQ) was administered to 211 patients with rheumatoid arthritis (RA) and 107 patients with osteoarthritis (OA) participating in five drug trials of NSAIDs at the beginning of the studies, and was commented upon by 17 clinical rheumatologists.

Results-Factor analysis of 211 patients with RA produced a unique factor solution. RA patients were more preoccupied with their illness and its effects and worried more about their health than patients with OA. Patients who withdrew from drug trials showed behaviour patterns similar to those of chronic pain patients, and different from those of patients who completed the studies. When asked to account for a rheumatoid patient's response to the IBQ, rheumatologists focused on physical symptoms and did not recognise some of the psychological issues which patients saw as being relevant.

Conclusions-We have demonstrated differences in illness behaviour between patients with OA and with RA. Patients withdrawing from drug trials of NSAIDs showed differences in illness behaviour compared with those successfully completing the trials. Rheumatologists underestimated the impact of the disease on their RA patients' psychological well being.

\section{(Ann Rheum Dis 1995; 54: 245-250)}

Patients with arthritis have a variety of patterns of coping and behavioural adjustment to the threat and suffering caused by their disease. ${ }^{12}$ These patterns may be reflected in compliance with medication regimens and involvement in a variety of treatments, ${ }^{34}$ and may thus be important predictors of outcome because of their impact on treatment compliance and level of disability. ${ }^{12}$ These characteristics are seldom discussed when considering the acceptance and resultant outcomes of physical and pharmacological treatments. This is an important issue when interpreting the results of drug trials, as there has been little investigation of the role of psychological factors influencing patient intolerance of medication independent of the direct pharmacological effects.

It is known that many patients withdraw from drug trials for poorly defined reasons. This phenomenon requires more investigation as it may simply not be an indicator of assumed toxicity. Furthermore, consideration of the selective factors determining who completes drug trials raises the issue of the extent to which these results can be generalised to clinical settings. Previous research has shown that a major factor in patients accepting and remaining on drugs is related to their attitudes and beliefs about their health and disease. ${ }^{3}$

There are a range of psychological and behavioural variables which can influence and describe adjustment in arthritis, such as personality and coping style, psychological symptoms, and patterns of illness appraisal. ${ }^{12}$ The concept of illness behaviour has been derived to describe the pattern of response to symptoms and the characteristic attitudes of patients along a series of dimensions. ${ }^{5}$ This phenomenon has been chosen for investigation in this study because it is a useful and parsimonious perspective in clinical settings. It considers the relationship between the severity of the disease process and the appropriateness of the extent of disability. ${ }^{1}$ Illness behaviour also describes the attitudes and behaviour of patients in the clinical setting which appear to be important elements of the adaptational process and can be measured using a questionnaire. ${ }^{5}$ It is important to emphasise that illness behaviour is a construct which overlaps with other dimensions of psychological functioning such as coping, beliefs, and attitudes, which can be measured independently. However, illness behaviour provides a clinically useful perspective that has a common sense utility.

One important question is if there are illness specific patterns of behaviour in arthritis in contrast to other general patterns of response. To date, no study has specifically examined the unique patterns of illness behaviour in rheumatoid arthritis, although there are likely to be significant variations in the patterns of adjustment. In other patient populations significant differences in patterns of illness behaviour have been observed-for example in patients with chronic pain, patients with myocardial infarction, and general practice

Bedford Park

South Australia 5042.

Accepted for publication 25 October 1994 
attenders. ${ }^{5}$ Patterns of adjustment characterised by a lack of acknowledgment of the emotional dilemmas caused by illness, high levels of preoccupation with physical distress and illness related fears tend to predict poor treatment response and are often seen in chronic pain patients. A questionnaire designed to measure illness behaviour, the illness behaviour questionnaire (IBQ), was developed in chronic pain patients. Its factor structure is frequently applied to the study of illness behaviour in a range of disease groups, with little question of its applicability. Therefore in this study we wished to investigate the relative importance of the illness specific patterns of behaviour, in contrast with the maladaptive attitudes and behaviour seen in many pain clinic patients, as predictors of maintenance of participation in drug trials. Examining such factors in drug trials is important to test the adequacy of the outcome measures agreed at the OMERACT Conference, Maastricht, ${ }^{6}$ which exclude any consideration of this important behavioural and attitudinal dimension. These measures exclusively focus on the physical dimensions of the disease process and the disability observed and are likely to provide an incomplete picture of factors which influence completion of a drug trial.

Against this background, this study aimed to examine a series of questions:

(1) Are there specific patterns of illness behaviour identified in patients with arthritis? A subsidiary issue is if there are different patterns of illness behaviour in rheumatoid arthritis (RA) and osteoarthritis (OA). This is an issue because one disease is a systemic disease which poses different tasks of adjustment as it tends to occur earlier in life and can have a more destructive and unpredictable course. We also wished to identify those aspects of illness behaviour which rheumatologists would characterise as being strongly related to the disease process, to assist in the interpretation of the patterns identified.

(2) Is illness behaviour associated with withdrawal from drug trials for poorly defined reasons? In particular, does the pattern of behaviour associated with chronic pain better predict withdrawal than the patterns of illness behaviour characteristic of arthritis?

\section{Patients and methods}

The patient population consisted of:

(1) Two hundred and eleven patients with rheumatoid arthritis (RA) attending three referral centres:

(a) One hundred and eight patients attending two suburban teaching hospitals in Adelaide. Their ages ranged from 44 to 81 (mean 66.4 , SD 7.9 ) years. They had a mean disease duration of $12 \cdot 2$ (SD $11 \cdot 3$ ) years. Their mean (SD) pain score using visual analogue scales was $48.3(23.6) \mathrm{mm}$. Fifty one percent of these patients had other illnesses, usually ischaemic heart disease or chronic obstructive airways disease. Sixty one patients were taking a disease modifying antirheumatic drug or prednisolone. (b) Thirty seven patients with early RA (less than two years) from Adelaide. Ages ranged from 31 to 74 (mean 52.5, SD 13.8) years.

(c) Sixty six patients attending a teaching hospital in Sydney. Ages ranged from 18 to 64 (mean 41.8 , SD $10 \cdot 0$ ) years. They had a mean disease duration of 1.5 (range $0-5$, SD $2 \cdot 0$ ) years.

(2) One hundred and seven patients with primary generalised $\mathrm{OA}$ attending clinics in Adelaide. Ages ranged from 46 to 86 (mean $70 \cdot 1$, SD $7 \cdot 1$ ) years. Their mean pain score was 46.9 (SD 18.2) $\mathrm{mm}$. Seventy five percent of the OA patients had comorbidities with the same disease spectra as the 108 patients with RA.

Patients with RA fulfilled the 1987 revised criteria of the American College of Rheumatology. ${ }^{7}$ Patients with OA had clinical or radiological OA affecting three or more groups of joints, as described by Kellgren and Moore. ${ }^{8}$

The illness behaviour profile is based on responses to a questionnaire derived by Pilowsky. ${ }^{5}$ The IBQ is a 62 item pencil and paper questionnaire with a yes/no format. It is a measure of attitudes to illness and illness behaviour and consists of seven factors: general hypochondriasis, disease conviction, psychological perception of illness, inhibition of affect, affective disturbance, denial, and irritability. Disease conviction is characterised by affirmation that physical disease exists, symptom preoccupation and rejection of the doctors' reassurances. These seven dimensions have been derived after analysing the responses to the questionnaire from 100 consecutive patients referred for management of intractable pain to the pain clinic at a large metropolitan hospital. Reliability and validity have been evaluated. Major factor analytic studies have also been performed with groups of patients with myocardial infarction, general practice attenders, and patients awaiting coronary artery bypass surgery. ${ }^{5}$

Factor analysis is a technique which is designed to reveal whether or not the pattern of responses on a number of tests or items in a questionnaire can be explained by a smaller number of underlying traits or factors. ${ }^{9}$ It can be used to indicate if the various items on the questionnaire can be grouped into a few clusters with each cluster reflecting a different construct.

The impact of illness behaviour on completion of drug trials was examined in the first group of 108 patients with RA and the 107 patients with OA. They were participating in five drug trials assessing the efficacy and toxicity of three non-steroidal anti-inflammatory agents (NSAIDs) (ketoprofen, ketoprofen SR and sulindac). These were conducted over six months and the IBQ was administered at entry to the studies. The patients completed the questionnaire at home and returned it at the time of their next appointment or by mail. The response rate was $100 \%$.

Patients were classified according to whether they completed these studies or withdrew for a variety of side effects.

To ascertain the items which might be strongly determined by the disease process in 
$\mathrm{RA}$, the questionnaire was sent to 20 practising rheumatologists. They were asked to identify those items among the 62 which would be associated with predictable 'yes' or 'no' responses by RA patients because of the presence of $R A$. Rheumatologists were instructed to leave blank those statements not predicted to be RA associated.

\section{STATISTICS}

The data were analysed using the Statistical Package for Social Sciences (SPSS-x) on the Flinders University of South Australia Encore Multimax Computer running system Unix BSD 4.2. Factor analysis of the 62 IBQ items of the patients with RA was performed. This group was used rather than the combined OA and RA population as this would have provided a factor solution which tended to minimise the differences between the groups. A confirmatory factor analysis could not be used as a comparative method because there were insufficient patients in the OA group to allow this approach. The responses obtained were factor analysed using principal component factoring with iteration, orthogonal rotation according to Kaiser's varimax criterion. The factors derived (RA factors) were chosen for Eigen values $>0.4$ for factors 1 to 6 and $>0.3$ for factors 7 and 8 . The means for each factor obtained from the patient groups were compared using a two tailed $t$ test. The Bonferroni adjustment for multiple comparisons was used.

\section{Results}

\section{FACTOR ANALYSIS}

There were no differences in responses to the IBQ questions between the three subgroups of patients with RA. Hence their responses were pooled.

Factor analysis of the 211 patients with RA produced a unique factor solution (table 1). The factor solution accounted for $32 \cdot 4 \%$ of the variance.

Factor 1 contained six items concerning illness vulnerability and accounted for $11 \cdot 8 \%$ of the variance. Factor 2 is concerned with the presence of life problems and is equivalent to scale 6 of Pilowsky analysis (denial). Factor 3 is characterised by illness preoccupation, factor 4 by dysphoria (and concerns the acknowledgment of anxiety and depression and is equivalent to factor 5 affective disturbance of the Pilowsky analysis), factor 5 by interfering pain, factor 6 by irritability (and is based on the degree of friction reported in social interactions); factor 7 is delineated by five items indicating a concern that others do not understand the significance of illness for the patients. Factor 8 has items dealing with the ability to communicate personal feelings.

RA COMPARED WITH OA

The 211 patients with RA and $107 \mathrm{OA}$ patients were compared using the Pilowsky and RA factors (table 2). These results show that the RA patients were generally more preoccupied with their illness and its effects and worried more about their health than the OA group. They more readily admit to other problems in their lives and they perceive psychological factors to play a more important role in their illness than do OA patients. However, they are not more depressed nor do they feel more vulnerable, and do not have more angry feelings and interpersonal friction, and have no more difficulties expressing personal feelings, especially negative ones, than do patients with OA.

\section{DRUG STUDIES}

Of the 107 patients with OA who participated in the trials, 64 completed the trial and 43 withdrew. In the 108 patients with RA, 71 completed and 37 withdrew. The reasons for withdrawal varied from well defined toxic reactions (for example gastrointestinal bleeding (three patients), skin rashes (one), and moderate to severe epigastric pain (four)) to poorly defined side effects such as vague abdominal pain (31), headaches (11), fatigue (four), malaise (five), light headedness (seven) and anorexia (seven). Seven patients had

Table 1 Results of factor analysis: eight factors have been specified

\begin{tabular}{|c|c|c|c|c|c|}
\hline Item No. & Nature of item & Loading & Item No. & Nature of item & Loading \\
\hline \multicolumn{3}{|c|}{ Factor 1: Upsetting vulnerability } & \multicolumn{3}{|c|}{ Factor 2: Other life problems } \\
\hline 44 & Worried about mind & $0 \cdot 70$ & 60 & Personal worries & $0 \cdot 84$ \\
\hline 15 & Upset with doctor & $0 \cdot 48$ & 27 & Life problems & $0 \cdot 80$ \\
\hline 38 & Worried about disease & 0.47 & 43 & Family problems & $0 \cdot 79$ \\
\hline 24 & More worried than most & 0.45 & 31 & Financial worries & $0 \cdot 56$ \\
\hline 6 & More prone to illness & 0.43 & $\star 46$ & Bad health biggest difficulty & $-0 \cdot 48$ \\
\hline 49 & Cooperative & $-0 \cdot 55$ & 55 & Worries over if fit & $-0 \cdot 43$ \\
\hline \multicolumn{3}{|c|}{ Factor 3: Illness preoccupation } & \multicolumn{3}{|c|}{ Factor 4: Dysphoria } \\
\hline 34 & Worried about possibility of serious illness & 0.66 & 18 & Easily anxious & 0.69 \\
\hline 2 & Something wrong with body & 0.62 & 47 & Easily sad & 0.60 \\
\hline 1 & Worried about health & $0 \cdot 48$ & 12 & Trouble with nerves & 0.60 \\
\hline 40 & Upset by face or body & $0 \cdot 41$ & 54 & Often depressed & 0.57 \\
\hline \multicolumn{3}{|c|}{ Factor 5: Interfering pain } & \multicolumn{3}{|c|}{ Factor 6: Irritability } \\
\hline 16 & Bothered by aches and pains & $0 \cdot 57$ & 51 & Easily angry & 0.60 \\
\hline 26 & Lot of illness pain & 0.51 & 61 & Lose patience & 0.58 \\
\hline 3 & Illness interferes with life & 0.51 & $\star 56$ & More irritable towards people & 0.44 \\
\hline$\star 46$ & Bad health biggest difficulty & 0.41 & & Easy to get on with & -0.51 \\
\hline \multicolumn{3}{|c|}{ Factor 7: Reaction of others } & \multicolumn{3}{|c|}{ Factor 8: Emotional restriction } \\
\hline 32 & Upset by way people take illness & $0 \cdot 38$ & & Angry/bottle up feelings & 0.58 \\
\hline 17 & Illness affects family/friends & 0.36 & 62 & Hard to show people personal feelings & 0.56 \\
\hline *56 & More irritable towards people & 0.33 & 53 & Prefer to keep feelings to self & 0.42 \\
\hline 39 & Illness not taken seriously & 0.32 & 22 & Easily express feelings & -0.44 \\
\hline 14 & Others realise what sickness is like & $-0 \cdot 47$ & & & \\
\hline
\end{tabular}

Item No. = IBQ question number; loading is for all patients with RA. ${ }^{\star}$ Note: Items 46 and 56 appear twice. 
Table 2 Comparison of patients with rheumatoid arthritis $(R A)$ and those with osteoarthritis $(O A)$

\begin{tabular}{llll}
\hline & $O A(n=107)$ & $R A(n=211)$ & $\mathrm{t}$ \\
\hline Pilowsky factors & & & \\
1. General hypochondriasis & $1.21(1.79)$ & $1.61(1.84)$ & 1.86 \\
2. Disease conviction & $2.68(1.71)$ & $2.85(1.56)$ & 0.88 \\
3. Psychological perception of illness & $0.84(1.02)$ & $1.27(1.20)$ & $3.13^{\star}$ \\
4. Affective inhibition & $2.57(1.40)$ & $2.71(1.43)$ & 0.85 \\
5. Affective disturbance & $2.45(1.68)$ & $2 \cdot 14(1.74)$ & 1.50 \\
6. Denial & $3.76(1.54)$ & $3.36(1.78)$ & 1.94 \\
7. Irritability & $1.47(1.60)$ & $1.54(1.54)$ & 0.41 \\
RA factors & & & \\
1. Upsetting vulnerability & $0.55(1.14)$ & $0.58(1.07)$ & 0.24 \\
2. Other life problems & $1.53(1.75)$ & $2.03(2.09)$ & 2.13 \\
3. Illness preoccupation & $0.94(1.18)$ & $1.53(1.36)$ & $3.799^{\star \star}$ \\
4. Dysphoria & $1.92(1.49)$ & $1.71(1.46)$ & 1.15 \\
5. Interfering pain & $2.83(1.32)$ & $2.52(1.32)$ & 2.01 \\
6. Irritability & $1.23(1.35)$ & $1.19(1.24)$ & 0.32 \\
7. Reaction of others & $1.03(1.29)$ & $1.10(1.24)$ & 0.48 \\
8. Emotional restriction & $2.09(1.25)$ & $2.16(1.28)$ & 0.45 \\
\hline
\end{tabular}

Values are mean (SD) where applicable. ${ }^{\star} \mathrm{p}<0.05 ;{ }^{\star} \mathrm{p}<0.01$ (corrected using the Bonferron method).

alteration of bowel habit (both constipation and loose bowel actions reported). Vague abdominal pain or dyspepsia occurred in $38.7 \%$ of patients (OA and $\mathrm{RA}$ ) who withdrew and $19.7 \%$ of patients who completed the studies.

Patients withdrawing from drug trials were compared with those completing them using both the Pilowsky and RA factor analyses. The RA and OA groups were analysed separately and the results are shown in tables 3 (RA) and $4(\mathrm{OA})$.

Significant differences were obtained using the Pilowsky factors but not the factors derived in the rheumatoid arthritis population.

Table 3 Comparison of drug trial participants: 108 rheumatoid arthritis (RA) patients

\begin{tabular}{|c|c|c|c|}
\hline & $\begin{array}{l}\text { Withdrawal } \\
(n=37)\end{array}$ & $\begin{array}{l}\text { Completed } \\
(n=71)\end{array}$ & $\mathrm{t}$ \\
\hline \multicolumn{4}{|l|}{ Pilowsky factors } \\
\hline 1. General hypochondriasis & $2 \cdot 0(2 \cdot 4)$ & $0.96(1.69)$ & $2 \cdot 82^{\star}$ \\
\hline 2. Disease conviction & $3 \cdot 19(1 \cdot 78)$ & $2.68(1.76)$ & 1.44 \\
\hline 3. Psychological perception & $0 \cdot 84(1 \cdot 12)$ & $0.85(1.09)$ & 0.03 \\
\hline 4. Affective inhibition & $2.76(1.52)$ & $2 \cdot 66(1.65)$ & $0 \cdot 29$ \\
\hline 5. Affective disturbance & $2.51(1.91)$ & $2.06(1.69)$ & $1 \cdot 28$ \\
\hline 6. Denial & $4 \cdot 11(1 \cdot 47)$ & $4.24(1.30)$ & $0 \cdot 48$ \\
\hline 7. Irritability & $1.38(1.64)$ & $0.99(1.45)$ & $1 \cdot 28$ \\
\hline \multicolumn{4}{|l|}{ RA factors } \\
\hline 1. Upsetting vulnerability & $0.81(1.31)$ & $0.41(1 \cdot 12)$ & $1 \cdot 67$ \\
\hline 2. Other life problems & $1.05(1.67)$ & $1.00(1.45)$ & $0 \cdot 17$ \\
\hline 3. Illness preoccupation & $1.70(1.27)$ & $1.23(1.29)$ & $1 \cdot 84$ \\
\hline 4. Dysphoria & $2.03(1.59)$ & $1.66(1.49)$ & $1 \cdot 18$ \\
\hline 5. Interfering pain & $3 \cdot 19(1 \cdot 18)$ & $2.83(1.37)$ & $1 \cdot 35$ \\
\hline 6. Irritability & $1.11(1.37)$ & $0.85(1.27)$ & 0.99 \\
\hline 7. Reaction of others & $1.00(1.29)$ & $0.87(1.10)$ & 0.54 \\
\hline 8. Emotional restriction & $2 \cdot 24(1.34)$ & $2 \cdot 14(1.39)$ & $0 \cdot 37$ \\
\hline
\end{tabular}

Values are mean $(\mathrm{SD}) .{ }^{\star} \mathrm{p}<0 \cdot 05$.

Table 4 Comparison of drug trial participants: 107 osteoarthritis patients

\begin{tabular}{|c|c|c|c|}
\hline & $\begin{array}{l}\text { Withdrawal } \\
(n=43)\end{array}$ & $\begin{array}{l}\text { Completed } \\
(n=64)\end{array}$ & $\mathrm{t}$ \\
\hline \multicolumn{4}{|l|}{ Pilowsky factors } \\
\hline 1. General hypochondriasis & $1.33(2.09)$ & $1.13(1.56)$ & 0.57 \\
\hline 2. Disease conviction & $3 \cdot 14(1 \cdot 86)$ & $2.38(1.55)$ & $2 \cdot 81^{\star}$ \\
\hline 3. Psychological perception & $0.95(1.09)$ & $0.77(0.97)$ & 0.93 \\
\hline 4. Affective inhibition & $2 \cdot 51(1.30)$ & $2.61(1.47)$ & $0 \cdot 35$ \\
\hline 5. Affective disturbance & $2.72(1.67)$ & $2 \cdot 27(1.68)$ & $1 \cdot 38$ \\
\hline 6. Denial & $3.35(1.74)$ & $4.03(1.33)$ & $2 \cdot 29^{\star}$ \\
\hline 7. Irritability & $1.70(1.68)$ & $1.31(1.54)$ & $1 \cdot 22$ \\
\hline \multicolumn{4}{|l|}{ RA factors } \\
\hline 1. Upsetting vulnerability & $0.63(1.42)$ & $0.50(0.93)$ & 0.57 \\
\hline 2. Other life problems & $1.95(2.02)$ & $1.25(1.49)$ & $2 \cdot 07$ \\
\hline 3. Illness preoccupation & $1 \cdot 02(1 \cdot 24)$ & $0.89(1.14)$ & 0.57 \\
\hline 4. Dysphoria & $2 \cdot 12(1 \cdot 52)$ & $1.78(1.46)$ & $1 \cdot 14$ \\
\hline 5. Interfering pain & $2.86(1.39)$ & $2 \cdot 81(1 \cdot 28)$ & $0 \cdot 18$ \\
\hline 6. Irritability & $1 \cdot 42(1.38)$ & $1 \cdot 11(1.32)$ & $1 \cdot 16$ \\
\hline 7. Reaction of others & $1.09(1.41)$ & $0.98(1.22)$ & 0.42 \\
\hline 8. Emotional restriction & $2.09(1.17)$ & $2.09(1.31)$ & 0.00 \\
\hline
\end{tabular}

Values are mean $(\mathrm{SD}) .{ }^{\star} \mathrm{p}<0.05$
The factors that seemed important differed between the RA and OA groups. In the RA group, the patients who withdrew during the drug trials scored significantly higher in general hypochondriasis. In the OA group the patients who withdrew scored higher for disease conviction, but lower for denial.

RHEUMATOLOGISTS' RESPONSES

Of the 20 questionnaires sent to rheumatologists, 17 were returned completed. Only seven items were predicted by more than $67 \%$ of rheumatologists as likely to be associated with typical responses in RA patients (table 5). Three of these items $(3,16,26)$ appear in RA factor 5 (interfering pain) and three $(1,2,40)$ in factor 3 (illness preoccupation). There was no consistent agreement amongst the rheumatologists: in only 21 of 62 items was there $100 \%$ concordance. The rheumatologists generally focused on the physical symptoms and did not recognise the anxieties, fears, or depression that could be equally ubiquitous (table 5).

\section{Discussion}

This study found that the patterns of illness behaviour described in a population of rheumatoid arthritis patients as measured by the IBQ were different from those seen in a chronic pain population. In particular, their response was characterised by a general sense of uncertainty about their physical and psychological health (factor 1). They also had perceptions of fear and preoccupation about their illness (factor 3) and other life stresses. This contrasts with the chronic pain patients, whose preoccupations are primarily hypochondriacal and about their physical ill health. This suggests that the factor structure which is normally used for the IBQ may not be appropriate for some investigations as it may not optimally characterise the patterns of illness behaviour in a population who do not suffer from intractable pain and attend a pain clinic.

When the pattern of illness behaviour was compared between RA and OA (table 2), the RA patients were more preoccupied with their illness and is effects and more readily admitted that psychological factors contributed to their illness. This pattern of illness behaviour characterises the uncertainties, fears, and psychological distress associated with a chronic unpredictable disease. ${ }^{10}$ OA patients, in contrast, were more preoccupied about somatic symptoms and their severity and focused on the notion that their illness was the only cause of their distress and problems in life.

The IBQ was then used to investigate the significant number of patients who withdrew from the drug trials for non-specific side effects. The reasons for withdrawal are important to investigate because they may affect the generalisability of the data. Withdrawal of the drug may be related to the attitudes and behaviour of the patients 
Table 5 Items in the illness behaviour questionnaire designated to be answered differently by patients with rheumatoid arthritis $(R A)$ because of the presence of $R A$ and responses of $R A$ patients

\begin{tabular}{rlll}
\hline $\begin{array}{l}I B Q \\
\text { item }\end{array}$ & Nature of item & $\begin{array}{l}\text { Rheumatologists } \\
\text { predicting } \\
\text { response } \\
(n=17)\end{array}$ & $\begin{array}{l}\text { RA patients } \\
\text { responding } \\
\text { true' }(\%) \\
(n=211)\end{array}$ \\
\hline 16 & Bothered by pains and aches & 100 & 82 \\
26 & Experience a lot of pain & 94 & 78 \\
3 & Illness interferes with life & 94 & 74 \\
40 & Upset by appearance of face or body & 82 & $33^{\star \star \star}$ \\
2 & Think that there is something seriously wrong with & 76 & 45 \\
25 & their body & 71 & 53 \\
1 & Illness affects sexual relations & 65 & 42 \\
12 & Worried about health & 0 & 30 \\
27 & Trouble with nerves & 0 & 24 \\
47 & Other life problems & 0 & 44 \\
22 & Easily sad & 0 & 35 \\
\hline
\end{tabular}

$\star \star \star p<0 \cdot 0005$. NB: For 62 comparisons, the $\mathrm{p}$ value chosen to identify statistical significance was 0.05 divided by 62 , with a resulting $p$ value of 0.0008 .

studied. ${ }^{3}{ }^{11}$ RA patients who withdrew tended to be more hypochondriacal (fearful and threatened by symptoms), while the OA patients who withdrew were more convinced that they had significant physical disease, were preoccupied by symptoms, and rejected the doctors' reassurances.

The RA factors were not significant in distinguishing patients withdrawing or completing the studies, which suggested that disease specific patterns did not predict withdrawal. In contrast, using the Pilowsky factors, derived from patients attending pain clinics, we demonstrated differences between the patients who withdrew from the drug trials and those who completed them. Thus patterns of illness behaviour typical of chronic pain predicted withdrawal. It makes sense that the patients with symptom preoccupation are the ones who will be unusually reactive to minor symptoms which arise when taking medications. The patients who completed the drug studies did have minor symptoms, but these did not prevent these patients completing the studies.

It is important to define the patients who withdraw from drug trials. Our findings are in agreement with those of Juby et $a l^{3}$ that patients with gastrointestinal symptoms attributed to NSAIDs had a significantly higher mean score for free floating anxiety, depression and somatisation than controls, suggesting that a patient's personality may influence the development of NSAID induced gastric symptoms.

Our results raise three questions which should be openly discussed. Whom should we select for drug trials? How do we generalise the findings of drug studies in selected patients to our clinic populations, given that toxicity is not the only predictor of withdrawal? ${ }^{11}$ Which symptoms are side effects of the drugs and which are related to behavioural responses? The responses of patients in the placebo arms of clinical trials of NSAIDs often show relatively high prevalence of gastric symptoms, suggesting that behavioural responses are as important as the toxicities caused by drugs. ${ }^{12} 13$ The findings of this study suggest that patients with a tendency to focus to an unusual degree on their symptoms are more likely to be intolerant of NSAIDs, yet these are often the patients whose symptoms are most difficult to manage. Caution must be used when the responses of such patients are predicted on the basis of existing clinical trial data. There is a need for specific data on the effectiveness of NSAIDs in these subpopulations.

When asked to predict the questionnaire item responses which were likely to be predicted by the disease process in RA, the rheumatologists underestimated the impact of the disease on their RA patients' sense of vulnerability to ill health, levels of depression, anxiety, and the distress the disease causes the patients when talking to doctors. The discrepancy between patients' experiences and the rehumatologists' perceptions highlights the need to consider the general consequences an illness has on the patient's life and not just the physical dimensions of the disease process. ${ }^{14-16}$ This raises questions about the quality of rheumatologists' interventions and advice when responding to patients about the general impact of the disease on their lives. It would seem that the focus on physical aspects of the disease process may not be fulfilling patients' needs. ${ }^{17}$ The discrepancy between patients' experiences and the rheumatologists' perceptions also raises the question whether such issues are adequately addressed in the training of doctors and rheumatologists. In a national survey of clinical rheumatology training of graduates of Australian medical schools, there was little evidence that students were exposed to the social dimensions of chronic illness; only $32 \%$ of students reported they had been shown how to assess a patient's psychological adjustment to illness. ${ }^{18} \mathrm{We}$ concluded that there were significant problems in the training of medical students in musculoskeletal disorders, particularly in relation to the assessment of disability and the appreciation of psychological factors.

The findings of this study need to be considered against the background of a series of methodological issues. The patients in this study had severe disease, as all were attending university hospital clinics. The RA and OA groups were not age or sex matched and hence the difference between the patients with RA and OA may be partially an effect of age. However, there were no differences in responses of the three subgroups with RA with different age ranges. Our study used only one instrument-the IBQ questionnaire. Ideally, these findings should have been validated using other instruments in the same group of patients. Juby et $a l^{3}$ have used the Middlesex Hospital Questionnaire only in patients taking NSAIDs, with results similar to ours. Our study was cross sectional and changes in responses to the IBQ may occur with time and disease stage and duration. However, $\mathrm{McF}$ arlane et al found no significant change in any of the scales of the IBQ over a three year period in a group of 30 patients with RA. ${ }^{15}$ Medical comorbidity, degree of disability and social demographic variables such as social support, social class, and educational background, may affect IBQ responses. Analysis of 
these important variables is beyond the scope of this paper.

Finally, our factor analysis accounted for only $32 \%$ of the total variance of the phenomena in this population. This implies that there are likely to be a variety of other patterns of illness behaviour superimposed on the disease specific patterns of adjustment. An important strength of this study is that it examined the factor structure of the IBQ in a larger population than that used in the derivation of the Pilowsky factors. It is also important to emphasise that in such studies the question always arises as to how the disease symptoms may modify the response to a questionnaire such as the IBQ, independently of the attitudinal and behavioural issues it aims to measure. The inclusion of somatic items in psychological measures has been an important source of methodological error in the study of the psychological adjustment in RA. ${ }^{19}$ In our study two populations with an arthritic process were compared and the differences emerged on items which were not simple measures of physical symptoms; rather, important attitudinal and behavioural dimensions were revealed.

In conclusion, we have demonstrated disease specific patterns of illness behaviour in patients with RA and OA. The withdrawal of patients from drug trials of NSAIDs, often with poorly defined toxicities, was associated with different patterns of illness behaviour than in patients completing the trials-an observation which complicates the interpretation and generalisability of these studies.

1 McFarlane A C, Brooks P M. Determinants of disability in rheumatoid arthritis. Br $\mathcal{F}$ Rheumatol 1988; 27: 7-14.
2 Manne S L, Zautra A J. Coping with arthritis: Current status and critique. Arthritis Rheum 1992; 35: 1273-80.

3 Juby A, Davis P. Psychological profiles of patients with upper gastrointestinal symptomatology induced by nonsteroidal anti-inflammatory drugs. Ann Rheum Dis 1991; 50: $211-3$.

4 Blalock S J, DeVellis B M, DeVellis R F, et al. Psychological well-being among people with recently diagnosed well-being among people with recently diagnosed rheumatoid arthritis. Do self-perceptions of abilities

5 Pilowsky I, Spence N. The manual of the illness behaviour questionnaire. Adelaide: University of Adelaide Press, 1981.

6 Conference on outcome measures in rheumatoid arthritis clinical trials, Maastricht, The Netherlands, 29 April-3 May 1992. F Rheumatol 1993; 20: 525-91.

7 Arnett F C, Edworthy S M, Black D A, et al. The American Rheumatism 1987 revised criteria for the classification of rheumatoid arthritis. Arthritis Rheum 1988; 31: 315-24.

8 Kellgren J H, Moore E. Generalised osteoarthritis and Heberden's nodes. BM7 1952; ii: $181-7$.

9 Streiner D L. Research methods in psychiatry. Figuring out factors: the use and misuse of factor analysis. Can 7 factors: the use and misuse
Psychiatry 1994; 30: 135-40.

10 Parker J C, Buchelew S P, Smarr K C, et al. Psychological screening in rheumatoid arthritis. $\mathcal{F}$ Rheumatol 1990; 17 1016-21.

11 Langley G B, Sheppeard H, Wigley R D. Placebo therapy in rheumatoid arthritis. Clin Exp Rheumatol 1983; 1 $17-21$.

12 Edwards W. Etodolac, aspirin and placebo in patients with rheumatoid arthritis: a 12-week study. Clin Ther 1983; 5: 495-503.

13 Delbarre F. Short-term study of indoprofen in comparison with placebo and indomethacin in rheumatoid arthritis. Eur f Rheumatol Inflamm 1981; 4:66-73.

14 Hawley D, Wolfe R. Anxiety and depression in patients with rheumatoid arthritis. A prospective study of 400 patients. 7 Rheumatol 1988; 15: 932-41.

15 McFarlane A, Brooks P. An analysis of the relationship between psychological morbidity and disease activity in rheumatoid arthritis. $\mathcal{F}$ Rheumatol 1988; 15: 926-31.

16 Parker J, Frank R, Beck N, et al. Pain in rheumatoid arthritis: relationship to demographic, medical and psychological factors. $\mathcal{F}$ Rheumatol $1988 ; 15$ : 433-7.

17 Hugglund K J, Haloy W E, Reveille J D, Alarseon G S. Predicting individual differences in pain and functional impairment among patients within rheumatoid arthritis. Arthritis Rheum 1989; 32: 851-8.

18 Crotty M, Ahern M J, McFarlane A C, Brooks P M. Clinical rheumatology training of Australian medical students. A rheumatology training of Australian medical students. A 119-20.

19 Pincus T, Callahan L F, Bradley L A, et al. Elevated MMP scores for hypochondriasis, depression, and hysteria in patients with rheumatoid arthritis reflect disease rather than psychological status. Arthritis Rheum 1986;29: 1456-66. 